\title{
Special issue of molecular diversity on "Al and ML for small molecule drug discovery in the big data era"
}

\author{
Kunal Roy ${ }^{1}$ \\ Published online: 19 July 2021 \\ (c) The Author(s), under exclusive licence to Springer Nature Switzerland AG 2021
}

The advancement and proliferation in artificial intelligence (AI) and machine learning (ML) algorithms have facilitated their applications in problem solving in many fields including drug discovery. AI/ML offers guidance to the research scientists to process, analyse, and understand the data, and their extensive application appears to be the future for drug discovery. The traditional drug discovery process is very costly and lengthy with limited success probability. The chemical space is very large, a fraction of which we have explored so far [1]. AI can be used to explore the chemical space and to understand the pattern of the complex big data. AI algorithms can make accurate predictions about complex systems involving the vast and unexplored space of molecules, reactions, and biological interactions. ML techniques have potential to identify new drug candidates in much less time than the conventional research [2-5]. However, the field is still young, having ample scope for improvements in the accuracy of AI algorithms and the adoption of more standardized and rigorous benchmarks so that the discipline can mature and improve further.

This collection on "AI and ML for Small Molecule Drug Discovery in the Big Data Era" showcases the latest developments in this field. There are twenty-two special issue articles giving an overview of machine learning approaches to drug discovery, evolving scenario of big data and artificial intelligence (AI) in drug discovery, applications of computational strategies in the field of functional foods, nutraceuticals and cosmeceuticals, recent trends in artificial intelligence-driven identification and development of anti-neurodegenerative therapeutic agents, and applications of convolutional neural networks (CNNs) in pharmacogenomics. The articles also present examples of various

Kunal Roy

kunal.roy.modi@gmail.com

1 Drug Theoretics and Cheminformatics (DTC) Laboratory, Department of Pharmaceutical Technology, Jadavpur University, Kolkata 700 032, India applications of machine learning tools including predicting blood-to-plasma concentration ratios of drugs from chemical structures and volumes of distribution in humans, a multi-conformational virtual screening approach based on machine learning targeting PI $3 \mathrm{~K} \gamma$, mutagenicity prediction using graph convolutional neural networks, application of tree-based machine learning models and molecular docking for molecular insights of ABL kinase activation, ensemble learning application to discover new trypanothione synthetase inhibitors, a novel artificial intelligence protocol to investigate potential leads for diabetes mellitus, in silico design of novel aptamers utilizing a hybrid method of machine learning and genetic algorithm, classification tasks related to drug safety with application of machine learning models, exploring novel DGAT1 inhibitors by combination of machine learning methods, pharmacophore model and 3D-QSAR models, identification of kinase inhibitors that rule out the CYP27B1 mediated activation of vitamin D by application of integrated machine learning and structurebased approaches, logistic matrix factorization and generative adversarial neural network-based method for predicting drug-target interactions, predicting HIV drug resistance using weighted machine learning method at target protein sequence-level, selection of potential inhibitors of acetylcholinesterase activity from SistematX (a natural products database) using machine learning methods, in silico prediction of chemical-induced hematotoxicity with machine learning and deep learning methods, and classification models and SAR analysis on CysLT1 receptor antagonists using machine learning algorithms.

$\mathrm{AI} / \mathrm{ML}$ in drug discovery is a fascinating area of research which has been identified as one of the thrust areas for Molecular Diversity. Several other manuscripts reporting results in this area are present in different stages of the manuscript handling system of this journal. There is an open invitation to authors to submit their research on this topic, and all the accepted papers will be published in the online topical collection on "AI and ML for Small Molecule Drug 
Discovery in the Big Data Era" (https://link.springer.com/ collections/ffiafcabac) in addition to their publication in regular issues. AI/ML being a hot topic, we hope that these papers will contribute significantly to further enhancing the impact of the journal. Of note that 2020 JCR impact factor has now reached to 2.943 (Clarivate Analytics) versus 2.013 in 2019.

Finally, I would like to thank all contributors for their fine work submitted to this journal with a request to continue submissions via the Editorial Manager system for future issues. For any query regarding the online topical issue, please feel free to contact me (kunal.roy.modi@gmail.com).

\section{References}

1. Lemonick S (2020) Exploring chemical space: can AI take us where no human has gone before? Chem Eng News 98(13):30-35

2. Zhavoronkov A, Ivanenkov YA, Aliper A, Veselov MS, Aladinskiy VA, Aladinskaya AV, Terentiev VA, Polykovskiy DA,
Kuznetsov MD, Asadulaev A, Volkov Y, Zholus A, Shayakhmetov RR, Zhebrak A, Minaeva LI, Zagribelnyy BA, Lee LH, Soll R, Madge D, Xing L, Guo T, Aspuru-Guzik A (2019) Deep learning enables rapid identification of potent DDR1 kinase inhibitors. Nat Biotechnol 37:1038-1040. https://doi.org/10.1038/ s41587-019-0224-X

3. Yang X, Wang Y, Byrne R, Schneider G, Yang S (2019) Concepts of artificial intelligence for computer-assisted drug discovery. Chem Rev 119:10520-10594. https://doi.org/10.1021/acs.chemr ev.8b00728

4. Réda C, Kaufmann E, Delahaye-Duriez A (2020) Machine learning applications in drug development. Comput Struct Biotechnol J 18:241-252. https://doi.org/10.1016/j.csbj.2019.12.006

5. Vamathevan J, Clark D, Czodrowski P, Dunham I, Ferran E, Lee G, Li B, Madabhushi A, Shah P, Spitzer M, Zhao S (2019) Applications of machine learning in drug discovery and development. Nat Rev Drug Discov 18(6):463-477. https://doi.org/10.1038/ s41573-019-0024-5

Publisher's Note Springer Nature remains neutral with regard to jurisdictional claims in published maps and institutional affiliations. 